\title{
Nationalismes corses et perspectives européennes
}

\section{Christian Lefébvre}

\section{(QpenEdition \\ Journals}

\section{Édition électronique}

URL : http://journals.openedition.org/conflits/656

DOI : $10.4000 /$ conflits.656

ISSN : $1777-5345$

Éditeur :

CCLS - Centre d'études sur les conflits lilberté et sécurité, L'Harmattan

\section{Édition imprimée}

Date de publication : 22 octobre 1992

ISSN : 1157-996X

Référence électronique

Christian Lefébvre, "Nationalismes corses et perspectives européennes », Cultures \& Conflits [En ligne], 07 | automne 1992, mis en ligne le 06 janvier 2003, consulté le 30 mars 2021. URL : http:// journals.openedition.org/conflits/656 ; DOI : https://doi.org/10.4000/conflits.656

Ce document a été généré automatiquement le 30 mars 2021.

Creative Commons License 


\title{
Nationalismes corses et perspectives européennes
}

\author{
Christian Lefébvre
}

Dimanche 20 septembre 1992 : à l'occasion du référendum sur la ratification par la France du Dimanche 20 septembre 1992 : à l'occasion du référendum sur la ratification par la France du traité de Maastricht, 56,72\% des suffrages exprimés en Corse ont été favorables au "non ", la Haute Corse arrivant en deuxième position des départements français ayant voté "non", la Corse du Sud en 9ème position. Le pourcentage d'abstention a été supérieur à $44 \%$. Déduire de ces résultats que la Corse est naturellement, à cause de son insularité, méfiante, voire hostile à l'idée de la construction européenne, serait une conclusion simpliste. L'objet de cette étude est d'analyser comment la construction européenne est perçue par les différentes organisations publiques et clandestines qui composent ce qu'on appellera par commodité de langage le mouvement nationaliste corse. On sera amené à constater que la place secondaire accordée par les nationalistes corses - comme par la plupart des autres grandes formations politiques insulaires ou métropolitaines - au débat européen et au traité de Maastricht n'est pas exclusivement le produit de moeurs insulaires spécifiques dont le caractère folklorique et caricatural constituerait en soi un mode explicatif. Le mouvement nationaliste corse, pris globalement, est désormais un acteur incontournable du champ politique de l'île, minoritaire mais non plus marginal. En ce sens, il est confronté à des questions qui ne sont pas toujours inhérentes à la Corse et relèvent de mécanismes dépassant une situation particulière, telles que la compétition politique dans un cadre institutionnel ou encore les logiques sociales de la clandestinité. Lors de la campagne électorale de septembre 1992, on a pu observer que la question de l'intégration européenne a suscité des prises de position divergentes à l'intérieur des grands partis politiques hexagonaux ou entre partis "alliés"; divergences présentées par leurs auteurs comme relevant de la parenthèse ponctuelle et ne remettant pas en cause les positions communes. Une "recomposition" du paysage politique français autour de ce thème étant par avance exclue, les prises de position en contradiction avec le choix du parti n'ont pas provoqué de scissions dans lesquelles 
l'enjeu européen aurait servi d'alibi à des rivalités internes pourtant réelles. Ce constat vaut également pour la classe politique corse, nationalistes inclus.

Un mouvement divisé

L'apparition du thème européen dans les discours et programmes des différentes organisations nationalistes a été tardif: évoqué de manière assez allusive dans des textes depuis le début des années 80 , il ne devient objet de réflexions et de propositions qu'à partir de 1987. Certes cette situation est banale; nombre de partis politiques hexagonaux sont dans le même cas. Seul le coup d'accélérateur donné en 1987 par l'Acte unique européen, avec la fixation de l'échéance quasi-mythique de 1993, a provoqué des interrogations nouvelles face à une question jusqu'alors sous-estimée. Mais il semble qu'un facteur supplémentaire ait joué dans le cas des nationalistes corses pour ralentir cette évolution : leur idéologie, leurs structures organisationnelles, leurs pratiques, reposent sur une vision des problèmes de l'île, structurée par le rapport de la Corse avec l'État français. L'évolution du mouvement lui-même; émergence de revendications régionalistes dans les années 60 , passage à des revendications de type nationaliste au début des années 70 , apparition d'un usage rationalisé de violence à partir de 1975, développement des structures dites de contre-pouvoirs à partir de $83 / 84$, éclatement en différentes organisations, tant publiques que clandestines, en 89/90, traduit une relation paradoxale avec l'État français. Référent permanent, l'État français est ressenti comme "responsable" de la situation dans l'île. Il est à la fois ennemi et interlocuteur obligatoire en tant que détenteur de pouvoirs qui lui sont contestés par les nationalistes. Or, un transfert partiel mais progressif de certaines prérogatives étatiques au profit d'institutions européennes ou locales, transfert qui devrait découler des mécanismes d'intégration communautaire, prendrait à contrepied plus de 20 années de lutte tournée exclusivement contre l'État français et ses relais politiques dans l'île, les "clans". Cette situation, même si elle est encore virtuelle, suppose pour les nationalistes corses la nécessité de procéder à des réorientations concernant leur propre fonctionnement et déborde du simple contenu politique du débat européen. Un bref rappel chronologique préalable est indispensable.

A l'heure actuelle, on compte 4 grandes organisations publiques nationalistes, plusieurs organisations politiques de taille réduite, un ensemble de syndicats sectoriels affiliés à un syndicat "généraliste" nationaliste, des structures de concertation internes ou externes au sein des organisations publiques, avec une structure à vocation globale (la Cunsulta Naziunale), une série d'associations nationalistes à vocations diverses (par exemple de soutien aux militants emprisonnés), et au moins 3 organisations clandestines principales, auxquelles s'ajoutent plusieurs groupes clandestins intermittents de faible taille. La distinction classique entre autonomistes et nationalistes remonte à la commission des premières actions violentes à caractère politique en Corse au début des années 70. L'ARC - Action régionaliste Corse, devenue en 1967 l'Action pour le renouveau de la Corse - est l'ancêtre des organisations publiques. Depuis 1977, l'UPC - Unione di u Populu Corsu - en est le prolongement et représente encore de nos jours la tendance autonomiste. Apparu en 1976, le FLNC Front national de libération de la Corse, dissous en 1983 et que l'on devrait pour plus de précisions nommer "ex-FLNC"-, produit de la fusion de plusieurs groupuscules clandestins, est l'organisation qui est à l'origine de la tendance nationaliste, ou pour être plus exact, de la tendance dite de la LLN - Lutte de libération nationale - inspirée par l'exemple des mouvements clandestins apparus au cours des vagues successives de décolonisation depuis 1945. Jusqu'en 1980, cette tendance n'avait aucune "façade 
politique légale", le FLNC diffusant lui-même des communiqués à la presse locale et des programmes politiques baptisés les "livres blancs". Ce vide fut comblé en 1980 par la création des $\mathrm{CCN}$ - Consultes des comités nationalistes - qui s'appelle aujourd'hui après des dissolutions successives, aCN - a Cuncolta Naziunalista -. Cette organisation publique peut être considérée comme le reflet fidèle des positions politiques défendues par le FLNC original. Le STC - Syndicat des travailleurs corses - est la structure d'impulsion de la stratégie dite de contre-pouvoirs à caractère social inaugurée en 1984. En octobre 1989, l'ANC - Accolta Naziunalista Corsa - est fondée après une scission au sein d'aCN. En novembre 1990, une deuxième scission au sein d'aCN est à l'origine de la création du MPA - Mouvement pour l'autodétermination -. Parallèlement, une scission se produit au sein du FLNC qui se divise alors en deux branches : le FLNC-canal historique, proche d'aCN et le FLNC-canal habituel, proche du MPA. II faut enfin noter l'apparition depuis la fin de 1989 d'un troisième groupe clandestin, bien que d'importance moindre : R-Rezistanza - et de groupuscules clandestins "thématiques", tels A Maffia No ! et A Droga Basta!

A l'occasion du vote du 20 septembre 92, le MPA avait appelé à voter "oui", l'UPC, aCN et i Verdi Corsi prônaient l'abstention alors que l'ANC, pourtant membre avec les trois formations citées précédemment de la coalition électorale Corsica Nazione, recommandait un vote négatif. Cet exemple récent de différences d'appréciation entre les principales organisations nationalistes publiques illustre assez bien le caractère instrumental de la question européenne pour elles. Les nuances des positionnements renvoient souvent à des considérations stratégiques, tactiques ou individuelles qui ne sont pas liées au contenu même du discours affiché, mais qui sont plutôt en relation avec le champ politique insulaire et surtout à des impératifs pratiques. En effet, depuis une quinzaine d'années, les différents mouvements nationalistes publiques et clandestins ont toujours développé l'essentiel de leurs activités autour de deux questions : d'une part, celle de la recherche des formes de lutte les plus appropriées contre l'État français dont on espère qu'elles permettraient de déboucher à terme sur une accession au pouvoir politique et économique dans l'île, et donc éventuellement sur une indépendance plus ou moins complète. D'autre part, celle des rapports internes au mouvement nationaliste, à la fois à l'intérieur de chaque organisation, mais surtout entre les différentes organisations le composant.

Si la dimension insulaire a toujours été privilégiée, ce n'est pas à cause d'une vision étriquée ou frileuse - par exemple le concept de "l'île-nation" -, mais parce que les nationalistes corses se sont toujours situés dans une logique de conquête du pouvoir politique. Ce postulat n'est pas sans conséquences, car actuellement la recherche d'une implantation électorale croissante, condition sine qua non d'un accès au contrôle politique dans l'île, est devenue une priorité absolue pour toutes les organisations nationalistes. Ce mécanisme de compétition politique n'est évidemment pas propre à la Corse. Or, obnubilés par la conquête de l'espace politique de l'île, les nationalistes corses ont longtemps négligé l'espace européen, sauf lorsqu'ils pouvaient espérer en tirer des avantages concrets et immédiats. De plus, l'idée que la construction européenne constitue en soi un obstacle à la souveraineté de l'île "pleine et entière", et donc à son indépendance potentielle perdure toujours. A contrario, la connexion ou plutôt la subordination à des revendications à caractère insulaire de questions en rapport avec l'Europe est mieux appréhendée par les organisations nationalistes ; dans cette situation, le caractère instrumental du thème européen peut être exploité au maximum. 
Une perception hétérogène de la construction européenne

L'examen de la production de discours totalement consacrés ou faisant simplement référence à la construction européenne met en relief la place limitée accordée à cette question par les nationalistes. En termes programmatiques stricto sensu, la dimension européenne est le plus souvent utilisée comme complément d'un concept politique plus important: l'indépendance. En revanche, les questions ponctuelles et pratiques susceptibles de toucher directement et rapidement la Corse font l'objet de développements plus poussés, voire spécialisés.

Partisans d'une solution institutionnelle dans le cadre de l'État français, l'UPC est l'organisation la plus engagée quant à l'insertion de la Corse dans la Communauté européenne. Ses propositions générales ${ }^{1}$ soulignent le besoin d'établir une liaison directe entre la Corse et Bruxelles, permettant de "casser le filtre de l'État français" et de participer directement à l'élaboration des programmes économiques d'aides aux régions les plus défavorisées qui peuvent concerner la Corse. Au mois d'août 1992, l'UPC formulait à propos des accords de Maastricht les interrogations suivantes: "La construction européenne affaiblit-elle le centralisme jacobin de l'État français? Renforce-t-elle la domination des États forts économiquement face aux régions périphériques défavorisées ?". L'UPC préconise également une ouverture vers le bassin méditerranéen au sens large, ainsi que vers les pays d'Europe Orientale, au nom de "la solidarité constante et réciproque avec toutes les minorités nationales d'Europe". Pour l'UPC, il faut que la "logique d'île" soit prise en considération par les institutions européennes seules à même d'imposer des dérogations aux normes européennes selon les autonomistes.

Le FLNC-canal historique, et donc aCN - rappelons qu'en principe le FLNC-canal historique est censé assurer la direction politique générale de la LLN en tant que structure politico-militaire hiérarchiquement supérieure, garante de l'orthodoxie idéologique nationaliste - sont partisans d'un "socialisme corse original" et développent une conception, fortement ancrée à gauche, de la construction européenne. Ainsi, le thème de l'exploitation économique des États du sud par ceux du nord est envisagé ${ }^{2}$ avec de longs développements sur les aspects économiques des rapports sociaux de production en Corse considérés comme facteurs d'aliénation du peuple. Ces deux organisations rejettent à la fois le régime capitaliste et les "dogmes afférents" et reprennent la notion d'autogestion à leur compte qui, dans un monde de mutation de la civilisation, doit s'accompagner de la conception d'une idéologie révolutionnaire, au sens de rupture mentale et sociale.". De même est refusée "l'Europe des marchands" dont l'objectif est "d'assigner à la Corse le rôle et la mission de centre de repos et de loisirs pour les privilégiés de la fortune.". Un extrait de la conférence de presse donnée par le FLNCcanal historique le 15 juillet 1992 résume assez bien l'extrême prudence formulée à l'égard de l'intégration européenne: "Nous suivons avec attention les évolutions évoquées par le traité de Maastricht, les thèses sur la subsidiarité et le début de reconnaissance des régions et des identités d'Europe". Nous tenons cependant à souligner les limites d'une telle entreprise : "Maastricht demeure une union d'États-bunker qui continuent à nier des nationalités et des peuples qui constituent son espace".

Le 8 septembre 1992, l'UPC, aCN et i Verdi Corsi donnaient dans une déclaration commune les raisons de leurs consignes d'abstention pour le référendum: "Nous demandons aux Corses de ne pas aller voter pour un scrutin pour lequel ils n'ont pas 
été consultés. L'analyse du traité montre clairement qu'aucune dérogation n'a été accordée pour la Corse, ni dans le domaine culturel, ni dans le domaine fiscal et économique, ni dans le domaine politique. Aucune reconnaissance du fait insulaire ne figure dans ce texte" ${ }^{13}$.

L'ANC a des positions sur l'Europe qui sont très proches de celles adoptées par aCN. La perception de l'intégration européenne y est cependant plus négative. L'ANC semble avoir une préférence pour des rapprochements fondés sur l'insularité en Méditerranée ou à proximité, en s'appuyant sur des cas existants (notamment Chypre, Malte, la Sardaigne et la Sicile). Elle constate ${ }^{4}$ que "la construction européenne nous permettra peut-être d'échapper au cadre de l'État-nation et de développer de nouveaux rapports avec nos voisins méditerranéens. Mais plus vraisemblablement, l'Acte unique confirmera, au niveau européen, les positions et les choix déjà acquis ne favorisant, en raison de l'inégalité de départ, que les plus forts économiquement". Dans un communiqué du 18 septembre 1992, l'ANC, tout en justifiant son appel à voter "non" par l'absence de reconnaissance de la notion de peuple corse par le traité lui-même et l'absence de dérogations pour l'île qui en résulte ajoute un élément politique original : "Enfin, se pose le problème du neutralisme. En effet, la politique de défense-commune implique I abandon de la neutralité. Ce grave problème se pose d'ores et déjà en Irlande". Rappelons que les divisions entre aCN et l'ANC portent officiellement sur le refus par cette dernière d'une hiérarchisation des structures de lutte et ses corollaires ; à savoir l'évolution vers un parti unique "infaillible" et la mise en place de structures bureaucratiques au sein des organisations de la LLN et ce, au détriment de pratiques de masse, c'est-à-dire démocratiques à l'égard de la base militante.

Les positions du MPA et du FLNC-canal habituel sont plus malaisées à cerner dans la mesure où, à notre connaissance, aucun texte à vocation programmatique n'a été encore publié. Partisan d une ouverture vers des partenaires n'appartenant pas à la famille nationaliste, le MPA était également favorable à des discussions avec l'État français lors de l'élaboration du deuxième statut particulier. Le 7 septembre 1992, le MPA, à l'issue de l'assemblée générale de ses délégués déclarait : "La question posée et le traité lui-même suscitent un certain nombre de réserves puisque la nation corse en est, pour l'instant, absente de par la volonté de l'État français. Le MPA oppose résolument au "non" le "oui" d'une dynamique européenne dont ce traité n'est qu'une étape. Au-delà de ce texte se dessine l'élaboration d'une Europe fédérale qui devra prendre en compte les réalités identitaires et nationales des peuples qui la composent.". Le MPA est pour l'instant la seule organisation nationaliste publique importante qui n'adhère pas à la structure de "coordination nationale" (synthèse) ni à la coalition Corsica Nazione. Il semble que le MPA, parti considéré comme modéré, éprouve encore quelques difficultés à établir un programme politique complet. L'objectif d'une indépendance totale est nuancé : dans sa conférence de presse du 24 septembre 1992, à la question "Certains mouvements nationalistes corses sont pour l'indépendance, d'autres parlent de souveraineté. Et vous ?", le FLNC-canal habituel donnait la réponse suivante: "Nous avons été les premiers au sein du nationalisme corse à affirmer la dimension européenne de la Corse. Notre adhésion à la construction d'une Europe des peuples implique un certain nombre de réajustements. Ainsi, il semble contradictoire de souhaiter une nouvelle construction politique remettant en cause les États-nations et de revendiquer par ailleurs l'avènement d'un nouvel État, futil corse. Nous revendiquons la souveraineté nationale corse dans l'interdépendance européenne". 
Enfin, le cas du groupe clandestin $\mathrm{R}$ ne pose lui aucun problème au niveau idéologique. Le 17 septembre 1991, dans l'unique conférence de presse qu'il a donnée à ce jour, R se définit comme une structure militaire qui n'a pas de vocation d'orientation politique : "Nous faisons quant à nous confiance à l'ensemble des organisations du mouvement nationaliste pour élaborer un plan de développement pour la Corse et son peuple. Nous ferons connaître éventuellement nos réflexions à ce sujet en apportant humblement notre contribution, si on nous le demande, notre volonté n'étant pas de nous substituer à l'ensemble des organisations publiques. Pour nous, elles sont les plus à même de porter loin cette réflexion et d'aboutir à une synthèse".

On peut observer, à partir de ce survol rapide des positions des organisations nationalistes, une constante: dans tous les discours, les nationalistes, lorsqu'ils envisagent les relations de la Corse avec ses voisins hésitent entre une perspective européenne et une perspective méditerranéenne. Cette dernière a été privilégiée jusqu'au milieu des années 80 en tant qu'espace géographique naturel de rattachement, alors que les discours actuels tendent à lier ces deux horizons en les présentant comme complémentaires, c'est-à-dire comme des éléments d'assise indispensables à une Corse souveraine et à terme indépendante. Un des attributs majeurs de la souveraineté réside dans la possibilité d'établir une diplomatie sur laquelle puisse s'appuyer une véritable politique étrangère. Malgré la volonté politique affichée par les organisations nationalistes de développer des relations internationales soit avec des peuples supposés être dans une situation comparables, soit avec les institutions communautaires ${ }^{5}$, force est de constater que l'établissement de relations avec des partenaires extérieurs à l'île en est toujours à un stade embryonnaire. Pour jouer un rôle international réel, les nationalistes doivent au préalable bénéficier d'une assise politique dans la population corse, aussi les activités liées à cette dimension internationale restent-elles par nature subordonnées à des facteurs politiques internes. C'est une des causes de la faiblesse du développement de structures tournées vers l'extérieur et donc d'un niveau d'activité restreint sur ce plan.

Une action internationale limitée

Actuellement, les regroupements entre les mouvements nationalistes européens, dont les corses, se retrouvent principalement à travers deux structures :

L'ALE - Alliance libre européenne -, fondée à Bastia en 1981 regroupe des représentants de six régions : l'Irlande, la Sardaigne, le Pays basque, les Flandres, l'Écosse et la Corse. Entre 1984 et 1989 l'ALE siégeait avec les Verts au Parlement européen au sein du groupe Arc en ciel. En effet, ces deux formations politiques se sont regroupées afin de pouvoir accéder au Parlement européen par le biais du scrutin proportionnel. Reçu en 1988 en Corse par les représentants de l'UPC, Antoine Waechter, leader des Verts français, avait sollicité le soutien des autonomistes en vue d'obtenir un appoint pour les parrainages de sa candidature à l'élection présidentielle. En échange l'UPC a obtenu une troisième place sur la liste des Verts aux élections européennes de 1989 ; c'est ainsi que le leader autonomiste Max Simeoni put être élu. Depuis 1989, le nouveau groupe Arc en Ciel présent au Parlement européen est constitué à partir du noyau des six députés de l'ALE. Cet "entrisme" institutionnel a eu relativement peu d'effets concrets, si on excepte le caractère symbolique d'une représentation de la Corse en termes nationalistes au niveau européen. L'activité de l'UPC à l'intérieur du Parlement de Strasbourg concerne surtout des dossiers ponctuels sur lesquels un accord entre les différents membres du groupe parlementaire ou les membres de formations "amies" 
est possible. Un exemple récent peut être donné par l'adoption par le Parlement européen le 16 septembre 1992 d'une résolution qui porte sur "les atteintes à l'environnement dues au rejet par les navires", à la suite du rapport Bertens. Le paragraphe 4 du texte comporte un amendement concernant le problème particulier du passage dangereux de pétroliers dans les bouches de Bonifacio, déposé par le député vert italien Gianfranco Amendora, amendement élaboré en collaboration avec Max Simeoni. On peut dire sans exagération que jusqu'à présent, le Parlement européen est resté un forum potentiel inexploité par les nationalistes corses ${ }^{6}$.

La CONSEO - Confédération des nations sans État de l'Europe Occidentale -. Il s'agit d'une structure non institutionnelle qui regroupe des mouvements nationalistes dont l'idéologie se rapproche fortement des thèmes révolutionnaires $(\mathrm{aCN}$, ANC dans une moindre mesure). En Corse, la tendance dite de la LLN entretient des relations qualifiées de privilégiées avec le Sinn Fein en Irlande et avec Herri Batasuna au Pays basque. Le problème est que si les points d'accord existent sur la condamnation des États "répressifs", le principe de coopération politique qui sous-tend l'existence de la CONSEO est battu en brèche par des divergences portant sur les moyens d'action à mettre en oeuvre et notamment sur la question de l'exercice de la violence politique au sein de la CEE. Certes, les contacts existent à travers les réunions et dans des colloques, par exemple les "journées internationales de Corte"7, des ébauches de projets sont discutées, des échanges culturels (groupes folkloriques) ou à caractère social (colonies de vacances) ont lieu, mais ici encore, à notre connaissance la dimension symbolique prime nettement sur les réalités organisationnelles.

On trouve également des associations proches des nationalistes corses mais qui sont spécialisées et ont une vocation à agir au niveau européen. Ainsi la délégation générale de la Corse de OEDHLF -Organisation européenne pour les droits de l'homme et ses libertés fondamentales -, spécialisée dans l'élaboration juridique de recours auprès des institutions européennes, a obtenu la condamnation de l'État français, prononcée le 27 août 1992 par la Cour européenne des droits de l'homme. Félix Tomasi, militant présumé du FLNC, ayant effectué 67 mois de détention préventive avant d'être remis en liberté par la cour d'assises de Bordeaux en avril 1988, recevra une indemnité d'un million de francs.

Flou idéologique, inefficacité relative des structures existantes, faible niveau d'activité : la dimension européenne externe à la Corse n'est pas, loin s'en faut, la préoccupation majeure des organisations nationalistes corses dont l'état d'impréparation semble patent dans ce domaine. A l'inverse, lorsqu'ils se situent dans une dimension interne à l'île, les nationalistes corses réussissent assez bien la greffe du thème européen sur certains problèmes spécifiquement corses.

La primauté de la dimension insulaire

D'un point de vue général, l'insertion de la construction européenne dans les discours nationalistes renvoie à une vision culturaliste à caractère ethnocentrique marqué. L'association d une logique d'insularité et d'un passé mythifié se traduit par la croyance de la prédisposition quasi naturelle du peuple corse à résister aux agressions ou aux menaces extérieures à l'île. Ces enseignements tirés de l'histoire de l'île tel que l'échec des invasions et des dominations successives (ou l'existence d'une courte période d'indépendance entre 1755 et 1769), confortent les nationalistes corses dans l'idée que la lutte contre tout corps étranger à l'île est le ciment du peuple corse. II s'agit, par ce biais, de susciter une prise de conscience de son identité par l'ensemble de la 
population. Par analogie, les nationalistes sont amenés à considérer que l'Europe est potentiellement une menace pour l'île, et doit être présentée à la population comme une puissance politique et économique à vocation dominatrice, au même titre que I'État français "colonisateur". D'autre part, la rhétorique révolutionnaire utilisée, audelà de ses aspects strictement idéologiques, renvoie également à une logique rationnelle de mobilisation de la population. Le travail des nationalistes sur ce plan consiste à agir de façon à ce que la population ne puisse s'imaginer pouvoir être défendue par quelqu'un d'autre que par eux. D'où l'affirmation qu'à terme, une entité politique nationale - au sens régionaliste du terme - à laquelle les Corses pourraient s'identifier doit s'imposer : il s'agirait d'une Corse pouvant maitriser seule son destin, c'est-à-dire souveraine et capable d'indépendance à l'égard des pressions extérieures à l'île, notamment dans le contexte européen.

L'importance de facteurs socio-culturels particuliers

Il est indispensable d'évoquer brièvement le poids de certaines logiques sociales sur le champ politique corse, dans la mesure où ces mécanismes qui déterminent les représentations et les comportements des nationalistes ont une influence certaine sur leurs perceptions des problèmes insulaires et donc sur les questions posées par l'intégration européenne. Deux notions étroitement imbriquées nous semblent particulièrement utiles à la compréhension du fonctionnement du système politique corse contemporain. Territorialité et clanisme ont une influence déterminante sur les pratiques de tous les acteurs politiques, y compris chez les nationalistes. Ces deux phénomènes existaient déjà lorsque la France annexa la Corse, mais la politique menée depuis par l'État français a fortement contribué à leur perpétuation sous des formes nouvelles adaptées à la situation institutionnelle de l'île et à ses aménagements successifs les plus récents.

La territorialité correspond à l'influence d'un acteur politique sur la population d'une zone géographiquement déterminée. Le degré de contrôle d'un territoire et de sa population renvoie à l'idée de "fief" politique, c'est-à-dire de zones dans lesquelles un acteur dominant peut disposer à sa convenance de la plus grande partie des ressources disponibles. Les moyens d'exercice d'une domination politique ne sont pas pris en compte ici. Le point important étant l'inscription territoriale du dominant à travers différents indicateurs ; par exemple les résultats électoraux obtenus pour un homme ou une organisation politique ou encore la possibilité d'exercer des prérogatives normalement dévolues à l'État ("impôt révolutionnaire") pour une organisation clandestine. En Corse, les expressions relevées dans la presse locale telles qu'"esprit pievan" - en référence à la pieve, regroupement traditionnel de plusieurs communes, souvent situées dans une même vallée, mais tombées de nos jours en désuétude avec les nouveaux découpages administratifs ou encore celle d'"archipel des influences", décrivent la réalité d'un morcellement géographique correspondant à une multitude d'implantations de pouvoirs locaux fondés sur la maîtrise des processus décisionnels politiques et économiques. Les détenteurs de cette puissance publique, qui sont normalement des relais exécutant au niveau micro-local les directives de leur hiérarchie administrative, ou exerçant les attributions qui leur sont confiées, lors des consultations électorales, sont guidés par des principes habituels de gestion des biens et ressources collectives. Ce découpage administratif fut imposé par l'État français, mais ne correspondait pas aux trois niveaux de référence traditionnels; la famille, le village et l'île elle-même. Cependant, il n'a fondamentalement pas remis en cause une perception traditionnelle, fortement culturaliste d'un espace politique naturellement 
fragmenté et conflictuel. L'implantation des organisations publiques nationalistes dans l'île n'est pas homogène et cette remarque vaut aussi pour les mouvements clandestins ; par exemple la Balagne, le Cortennais sont des zones considérées comme parmi les mieux "tenues" par les clandestins. A titre d'exemple, lors des dernières élections régionales de mars 1992, le MPA ${ }^{8}$ a obtenu 851 suffrages exprimés à Bastia, et 2560 à Ajaccio. De plus, l'implantation des nationalistes dépend aussi en partie de la distinction entre zones urbaines et rurales. Les scores réalisés par les nationalistes en zones rurales sont en général proportionnellement inférieurs à ceux obtenus dans les villes. On peut émettre l'hypothèse que le quadrillage de l'espace politique est plus délicat pour les nationalistes en zone rurale du fait du poids des élus traditionnels sur les espaces dans lesquels l'importance des réseaux familiaux est plus facile à maintenir. A contrario, les nationalistes, lorsqu'ils réussissent à s'implanter localement (sections dans les communes), reprennent des attitudes directement empruntées à la classe politique locale: Dans cette logique, la scission de l'ANC et d'aCN en 1989 pourrait n'être que l'illustration de la prédominance d'une majorité dans la région bastiaise à ce moment-là, et de la capacité d'une tendance dans le sud de l'île à s'organiser de façon autonome. Le terme générique de clanisme renvoie lui à un mode particulier de domination politique. Il est indissociable de la territorialité car il repose sur l'existence de réseaux d'influence superposés souvent fondés sur des clivages biparties, dont les activités s'inscrivent dans un cadre géographique délimité. La pratique claniste correspond à l'instrument de l'exercice d'une domination politique. Les clans tirent leur pouvoir de la relation de type clientéliste qu'ils ont su maintenir et faire évoluer depuis plusieurs siècles. L'originalité de ces pratiques repose sur la nature de l'échange de prestations entre le "patron" et son client: à la différence d'un engagement contractuel courant, la réalisation réciproque et entière de prestations préalablement définies ne suppose pas la fin de l'échange. Le client - la population ou un chef de clan de niveau inférieur, c'est-à-dire qui contrôle une zone d'influence moindre - accepte le principe d'une continuité de son soutien, de sa fidélité, en fait de son allégeance, à un patron même si l'espoir qu'il a d'une rétribution ultérieure est aléatoire faible voire quasi nul. La forme politique moderne du clanisme est fondée sur l'échange d'une promesse de vote par le client avec une rétribution par le patron qui peut prendre des formes multiples (de la délivrance d'un permis de construire à l'octroi d'une pension financière...). Le principe de rétribution sélective des clients par le détenteur effectif des moyens de distribution sur un territoire donné n'est pas fondé sur une délégation de pouvoirs consentie par une majorité de la population habitant sur ce territoire. Le fondement de la légitimité du clan repose sur l'acceptation par la population de la relation de dépendance qui la lie au clan à chaque niveau considéré. Le bien public n'est pas le bien collectif géré par le clan, qui rétribue exclusivement ses membres et ce au détriment des autres individus. C'est pourquoi un clan, quel que soit son niveau, n'a que rarement la possibilité d'exercer un contrôle total sur l'ensemble de la population d'un territoire clairement défini ; il y a presque toujours, structurellement, un espace pour un adversaire du même type.

Cette courte présentation $\mathrm{n}$ est pas exhaustive; en effet, la réalité contemporaine est plus complète dans la mesure où plusieurs types de clivages claniques peuvent se superposer, $\mathrm{v}$ compris sur une même zone de référence. L'État français utilisa - et utilise toujours les personnalités, les notables traditionnels comme un relais destiné à encadrer la population au niveau cantonal, communal, départemental et, depuis 10 ans, régional. En échange, l'État permit à ces notabilités de conserver une bonne partie de 
leur influence traditionnelle qui préexistait à son arrivée en Corse. Les nationalistes corses sont-ils touchés par ce phénomène ? Comme le remarque fort justement José Gil, les nationalistes ne savent toujours pas quelle réponse apporter la question: "le clanisme fait-il partie de l'identité corse ?". La dimension personnelle nous semble être un élément important: la "lutte des envies", la concurrence de personnalités, de "leaders", fondée non exclusivement sur la compétence mais sur le charisme, et qui est une des caractéristiques des pratiques claniques de la classe politique traditionnelle n'est pas absente chez les nationalistes. Les différentes scissions précédemment évoquées renvoient toutes, plus ou moins, à cette dimension couplée à d'autres facteurs de divergences. Ainsi, la querelle très vive entre $\mathrm{aCN}$ et le MPA masque Ie fait que les membres les plus connus (Alain Orsini, Dominique Bianchi) de la direction actuelle du MPA faisaient, jusqu'en 1990, partie de la direction collégiale d'aCN. Les accusations personnalisées réciproques que les dirigeants de ces deux formations se jettent à la figure sont révélatrices (enrichissement personnel issu d'une dérive mafieuse, compromissions avec l'État, "caste" d'élus institutionnels nationalistes tombés dans la "trappula", le piège du réformisme confortable...). Il en va de même pour le leader de l'ANC (Pierre Poggioli), ancien élu de l'assemblée territoriale issue du premier statut particulier dans le même groupe nationaliste. De la même manière, le retour "aux affaires", en tant que tête de liste de la coalition Corsica Nazione, d'Edmond Simeoni (absent de la scène publique depuis 1983), certainement le leader nationaliste historique, illustre le fait que les nationalistes sont parfaitement conscients de cette dimension et sont capables de l'utiliser rationnellement en tant qu'instrument de mobilisation. Dans la même optique, Léo Battesti, leader "nordiste" historique, récemment démissionnaire de la direction du MPA, a critiqué la stratégie majoritaire actuellement menée au sein du mouvement nationaliste corselz. Pour lui, la démarche populiste qui consiste à s'ériger en protecteur "contre la Mafia, les trafiquants, les spéculateurs, les magouilleurs, les clanistes..." est une dérive de nature claniste : "les habitants de ce pays sont en position d'assistés. Hier, on leur promettait des places, aujourd'hui, on leur promet un rempart pour les défendre !".

$\mathrm{Du}$ point de vue des logiques organisationnelles, la place secondaire accordée aux questions touchant l'Europe par les structures nationalistes politiques internes à l'île s'explique, outre leur antériorité chronologique, par une attitude pragmatique: les organisations ont désormais une expérience réelle du champ politique corse et de ses pratiques. Fonctionnellement, la montée en puissance de structures de type bureaucratique à l'intérieur des grandes organisations nationalistes est calquée sur une conception classique de la place des structures internes au sein des organisations politiques modernes: bureau exécutif, assemblée générale des délégués, découpage géographique en entités hiérarchisées par un système de représentation déléguée de type pyramidal. Le mode de fonctionnement des différentes organisations nationalistes internes les fait tendre à l'établissement d'une tutelle sur toutes les questions pouvant concerner de près ou de loin la Corse. La perspective européenne n'étant dans cette gestion des choix et activités qu'un secteur politique particulier ne justifiant pas organiquement de façon prioritaire la création d'entités externes spécialisées "autonomes". Les répercussions de cette logique bureaucratique de fonctionnement conduisent les différentes organisations nationalistes à n'aborder l'intégration européenne qu'à l'intérieur de créneaux sectoriels définis d'abord par rapport à l'île. On peut donner quelques exemples ponctuels de cette attitude relevés dans les 
pratiques des différentes organisations publiques ou clandestines - sans rentrer dans la polémique dont cette distinction fait l'objet.

Pour les organisations publiques

Les quatre grands partis nationalistes sont tous favorables à une participation aux institutions publiques en Corse, et ce à tous les niveaux qui font l'objet de consultations électorales. Mais les modes de scrutin utilisés ne sont pas tous, par leurs modalités techniques, également favorables à l'enracinement électoral des formations nationalistes. Ainsi le scrutin majoritaire à deux tours ou le suffrage indirect sont des facteurs de perpétuation d'une relative stabilité du personnel politique. Par contre, le scrutin proportionnel doit permettre une diversité plus large dans la représentation des sensibilités politiques minoritaires. En Corse, ce sont les deux assemblées à caractère régional issues des statuts particuliers de 1982 et 1991 qui sont sous cet angle les plus "abordables" en terme d'entrisme institutionnel par les partis nationalistes. D'ailleurs, une des raisons de la faillite de la première assemblée territoriale corse résida dans la difficulté à dégager une majorité stable et donc un exécutif fort à cause d'un trop grand émiettement des forces politiques représentées: majorité de circonstance et ralliements individuels parfois surprenants ${ }^{9}$ étant des critiques souvent formulées par les élus traditionnels corses à l'encontre du scrutin proportionnel. Le nouveau statut particulier consacré par la loi du 13 mai 1991 a tenté d'y remédier par des aménagements techniques : scrutin proportionnel mais à deux tours avec impératif d'obtention de $5 \%$ de suffrages exprimés au premier tour pour accéder au second, prime de trois sièges (pour un total final de 51) pour la liste arrivée en tête à l'issue du deuxième tour, refonte des listes électorales ${ }^{10}$, conseil exécutif distinct des élus de l'assemblée dont il provient. Parallèlement, l'assemblée de Corse est l'institution qui est la plus proche, par son principe, des aspirations politiques des nationalistes. Les compétences qui lui sont dévolues ou transférées par l'État constituent théoriquement des moyens d'action conséquents au niveau régional ${ }^{11}$. L'assemblée de Corse représente plus qu'une tribune officielle qui ferait office de simple instrument de propagande médiatisée pour les nationalistes. Ces derniers l'envisagent comme une institution susceptible, si l'État français lui en donne effectivement les moyens, d'assurer la prise en charge d'une phase de transition caractérisée par un accroissement d'autonomie politique et économique de l'île vis-à-vis de la métropole (ceci dans l'objectif de l'exercice d'un droit à l'autodétermination) dans des secteurs auparavant supervisés par l'État français. Lors du deuxième tour des élections régionales de mars 1992, la coalition Corsica Nazione a obtenu 16,85\% des suffrages exprimés (soit 9 élus pour 21 872 voix), le MPA atteignant 7,98\% des suffrages exprimés (soit quatre élus pour 10360 voix). Devenue la deuxième force politique de l'assemblée en réunissant près du quart des suffrages exprimés, les partis nationalistes ont recentré leurs activités dans, ou autour, de cette institution, leur position étant déterminée au cas par cas en fonction des thèmes abordés par l'assemblée. Le dépôt d'amendements ou de motions commence à atteindre un "rythme de croisière", car confrontés à des débats sectoriels, les partis nationalistes peuvent affiner leur position en leur donnant une portée concrète et détaillée. L'aspect européen est perçu par eux soit comme un atout, soit comme une menace pour l'île; cette formulation en termes d'avantages ou d'inconvénients est facilement observable dans le cadre de l'assemblée de Corse.

Ainsi, sur un plan politique, les nationalistes voient d'un oeil bienveillant - bien que teinté de scepticisme - la déclaration d'intentions du Parlement européen sous la forme d'une charte communautaire de la régionalisation européenne adoptée en annexe de la 
résolution sur la politique régionale communautaire et l'Europe des régions, le 18 novembre 1988. Le MPA a déposé une motion demandant "la mise en place d'une représentation permanente de la Corse à Bruxelles" à la fin du mois de juillet 1992, qui a abouti, après amendement, au vote d'une motion par l'assemblée de Corse qui propose "la mise en place d'un correspondant (et non pas d'un représentant) permanent de la collectivité territoriale à Bruxelles pour la préparation et le suivi des mesures et des programmes concernant la Corse". En revanche, le principe de subsidiarité entraînant des relations directes avec Bruxelles peut se révéler à double tranchant, du point de vue des nationalistes, la CEE pouvant être tentée par un dirigisme dans des domaines relevant de sa compétence exclusive qui auraient des répercussions sur l'île ou si les objectifs de l'action qu'elle envisage ne pouvaient pas être atteints par I'État français.

De même, sur un plan économique et social, l'appréciation de l'impact en Corse du rôle de la CEE s'exprime dans une stratégie à double versant: jouer la CEE contre l'État français ou l'inverse pour servir au mieux les intérêts insulaires. Par exemple, la recherche de maintien de la Corse dans "l'objectif $n^{\circ} 1$ " au titre de l'aide financière que la CEE apporte aux régions en retard de développement à travers les différents programmes d'aides sectorielles de deuxième génération ${ }^{12}$ est à mettre en perspective avec les tentatives d'obtention d'un statut fiscal dérogatoire au droit européen pour la Corse. Ces efforts pour tenter d'obliger l'État français à négocier avec Bruxelles un statut particulier protégeant l'économie corse de l'ouverture des frontières ont lieu dans le cadre de la commission mixte État-collectivité territoriale qui est destinée à l'élaboration concertée et préalable du dossier fiscal de la Corse vis-à-vis de Bruxelles, mais aussi de Paris $^{13}$. II s'agit de continuer à bénéficier, après l'échéance du 1er janvier 1993 , de la manne européenne, tout en préservant les avantages acquis grâce à l'État français, la difficulté paradoxale étant de parvenir à un équilibre en ce sens. En résumant, on peut dire que trop d'aides d'origines communautaires pourraient rendre injustifiées les aides indirectes accordées à l'île par l'État français en matière fiscale, au titre du handicap lié à l'insularité et du principe de continuité territoriale, mais qu'un statut fiscal dérogatoire trop protectionniste pourrait rendre la Corse trop riche pour prétendre bénéficier des programmes d'aides aux régions défavorisées de la CEE. De même, la demande faite à l'État français d'élaborer un code des investissements est destinée à éviter un accaparement spéculatif de l'économie de l'île par des capitaux extérieurs, la Corse risquant d'être exclue des retombées financières d'un afflux de capitaux non maîtrisé. Pour les nationalistes corses, cette protection de l'économie insulaire passe par l'établissement obligatoire de principes d'économie mixte qui seuls peuvent garantir un contrôle des prises de participation étrangères et la réaffectation d'une partie des profits dégagés à des investissements productifs dans et pour l'île.

Enfin, sur un plan culturel, les nationalistes sont très intéressés par la résolution 192 dénommée charte européenne des langues régionales ou minoritaires, adoptée le ?2 juin 1992 par le comité des ministres du Conseil de l'Europe (instance qui, avec ses 36 membres, déborde largement du cadre de la CEE). Corsica Nazione souhaite que l'assemblée de Corse présente, dans le cadre de l'article 26 du statut de 1991, une motion en ce sens auprès de l'Assemblée nationale française pour faire pression sur l'État, toujours très réticent à ratifier un acte qui pourrait déboucher sur une coofficialité des langues française et corse ${ }^{14}$. L'objectif est simple: la reconnaissance officielle d'une langue rend difficile la négation de l'existence du peuple qui l'utiliseS Le 26 juin dernier, Corsica Nazione avait déposé à l'assemblée de Corse une motion, rejetée 
par 23 voix contre 22, qui concernait entre autres cette question de l'officialisation de la langue corse et son corollaire, la prise en charge de son enseignement. Dans cet exemple, les institutions sont envisagées comme des moyens de pression utilisables cantre l'État à partir d'une revendication insulaire particulière jusqu'à présent bloquée. La langue corse est dans cette bataille un vecteur de communication symbolique très utile aux nationalistes en liant les notions de langue et de corsitude. Les nationalistes peuvent prétendre au monopole de la défense de l'identité culturelle corse, à la fois visà-vis de l'État français - langue dominante - et de la CEE - uniformisation linguistique liée à une intégration excessive. En revanche, l'aspect menaçant de la CEE pour les nationalistes se retrouve dans leur demande d'élaboration d'un schéma d'aménagement du territoire, spécialement dans les zones littorales et dans l'arrière pays "sauvage". Cette démarche correspond à un souci de protection du patrimoine écologique et historique de l'île, menacée par les principes de libre circulation des personnes et des capitaux ("bétonnisation" anarchique liée au développement du tourisme).

... comme pour les organisations clandestines

Les organisations clandestines, elles, ont intégré la question européenne dans leur pratique rationalisée de la violence. Rappelons qu'en Corse, les méthodes utilisées sont rodées depuis plus de quinze ans. Ce sont en grande majorité des attentats à l'explosif contre des biens publics ou privés (les homicides à caractère politique étant très rares) comportant une dimension symbolique avérée: la nature des cibles visées est théoriquement sans équivoque, les clandestins ayant le souci d'éviter de tendre une "perche" à l'État français en lui fournissant les arguments d'une politique de criminalisation des attentats, fondée sur un amalgame avec la délinquance de droit commun. Bien qu'officiellement respectueux d'une trêve globale - que l'on a qualifiée de perlée - des actions militaires, plus ou moins bien respectée depuis la fin de 1990, les deux FLNC ont précisé à plusieurs reprises qu'ils ne s'interdisaient pas des actions à caractère ponctuel ou politico-militaires (forte charge symbolique). Sur ce créneau de l'activisme violent, la dimension européenne ou internationale est présente par le biais de la spéculation immobilière d'origine étrangère, de la dérive mafieuse et du trafic de drogue. Elle est rarement directement évoquée dans les revendications: drogue, blanchiment de capitaux privés d'origine douteuse sont présentés comme des conséquences prévisibles et déjà perceptibles de l'ouverture des frontières. La concurrence entre organisations clandestines ne se traduit pas sur ce thème par une surenchère violente et incontrôlée, chaque organisation souhaitant surtout faire la preuve de ses capacités d'analyse et d'action par rapport à ses concurrents. Certains attentats explicitement liés à ces thèmes, comme ceux revendiqués par "A Droga Basta" ou "A Mafia No" sont d'une imputation précise malaisée : les revendications évoquées précédemment sont-elles le fruit de groupuscules réellement autonomes ou d'un découplage pratique permettant aux deux FLNC de se dissocier d'actions dont le caractère politique est parfois équivoque ${ }^{15}$. La même remarque vaut également pour $\mathrm{R}$, bien que les attentats nécessitant un certain degré de professionnalisme et des moyens importants permettent de supposer au moins un minimum d'autonomie fonctionnelle de ce groupe armé (comme le montre le plasticage en plein jour le 26 juillet 1992, par un commando en hélicoptère, d'une vingtaine d'appartements sur l'île privée de Cavallo, action explicitement revendiquée au nom de "la lutte contre les intérêts de la Mafia de Cavallo"). Le caractère extérieur à l'île de la menace risque d'aboutir à une radicalisation d'une partie des clandestins, ce qui aurait pour conséquence l'utilisation 
de moyens d'action (comme les homicides) considérés comme "légitimes" pour faire face à cette agression venant de l'extérieur. En 1986, l'exécution par le FLNC de deux trafiquants de drogue originaires du Maghreb avait suscité de nombreuses polémiques, y compris à l'intérieur même des différentes organisations nationalistes, ce qui avait provoqué un coup d'arrêt à la campagne antidrogue alors en cours, du moins dans ses effets les plus extrêmes. L'actualité récente semble indiquer que ce type de débat n'est pas caduc, le FLNC-canal historique ayant à la mi-octobre 1992 implicitement reconnu son implication directe dans l'élimination physique de plusieurs trafiquants, dont certains étaient d'origine corse (il n'a cependant pas été jusqu'à préciser les noms de ses victimes). Le caractère importé de la menace dans l'île couplé à une dimension de droit commun est perçu par certains clandestins comme une dérogation possible à l'autolimitation de la violence pratiquée ordinairement. Elle pourrait justifier les homicides refusés jusque-là, car les clandestins, depuis la création du FLNC, ne s'étaient pas investis de façon significative dans la lutte contre le milieu insulaire traditionnel. L'internationalisation de ce milieu, la perte progressive de sa corsitude ("code" de conduite entre truands corses) et surtout de son caractère structuré et hiérarchisé (disparition des "parrains" locaux trop voyants) qui entraîne la multiplication de bandes rivales pourraient remettre en cause l'attitude passive des clandestins à l'égard de la délinquance de droit commun dans l'île - l'éventualité de représailles mutuelles risquant de dégénérer de façon incontrôlée s'étant avérée jusqu'à présent assez dissuasive. C'est un des enjeux du thème de la "dérive mafieuse" de l'île : une Mafia insulaire ou une Mafia importée d'Italie ou d'ailleurs n'implique pas nécessairement le même type de retenue pour "traiter ce problème" de la part des clandestins.

Zone périphérique économiquement pénalisée, la Corse peut espérer beaucoup de la politique de régionalisation de la CEE. Mais le retard accumulé dans la perception politique de l'émergence du fait européen implique une remise en cause profonde des comportements de l'ensemble de la classe politique de l'île, nationalistes inclus. Les difficultés rencontrées de ce point de vue tant au niveau institutionnel qu'au niveau des stratégies des forces politiques sont toujours un obstacle majeur à une insertion non conflictuelle dans l'espace communautaire, surtout, tant que ce dernier ne sera envisagé que sous la forme d'une contrainte politique et économique extérieure à l'île supplémentaire et non comme une association potentiellement symbiotique.

Bibliographie

Ouvrages

Accolta Naziunali Corsa (ANC), Pruposti Pulitichi (document en supplément au journal A FIARA), juin 1990, 285 p. (bilingue français/corse).

BINDI Ange-Laurent, Le naufrage de l'autonomisme corse 1982-1987, L'Harmattan, mai 1990, $140 \mathrm{p}$.

BINDI Ange-Laurent, Autonomisme lutte d'émancipation en Corse et ailleurs 1984-1989, L'Harmattan,juin 1990, 315 p.

BINDI Ange-Laurent, La Corse - autour, L'Harmattan, septembre 1992, 200 p.

Bonnardi Fabrice, Corse. La croisée des chemins, L'Harmattan, septembre 1989, 118 p.

A Chajma pa l'indepindenza e u sucialismu, Ghjustifcazione di l'indepindenzu di a Corsa, A Chajma, février 1989 (document photocopié).

Corsica Informazione,"Secondu Quoternu", supplément à U Ribombu, publication irrégulière (en particulier le numéro 12 de mars 1991). 
Culioli Gabriel-Xavier, Le complexe corse, Gallimard, collection Au vif du sujet, juillet 1990, $282 \mathrm{p}$.

Délégation corse de l'organisatíon européenne des droits de l'homme et pour ses libertés fondamentales (OEDHLF), La Corse, l'Europe et le droit, La Marge, juillet 1991, $100 \mathrm{p}$.

Gil José, La Corse entre la liberté et la terreur. Etude sur la dynamique des systèmes politiques corses, La Différence (2ème éd. revue et augmentée), mai 1991, 278 p.

Labro Michel, La question corse, Entente, 1977, I 71 p.

Poizat-Costa Marie-France, Le problème corse. Essai d'anthropologie philosophique, L'Harmattan, février 1987, 183 p.

Poli Moune, La Corse au poing. L'implosion d'une société bloquée, L'Harmattan, avril 1991, 234 p.

Renucci Jeanine, La Corse, PUF, coll. Que sais-je ?, avril 1987, 126 p.

Stagnara Vincent, Minorités et statuts. L 'exemple de la Corse. Regard sur l'Europe. Problémalique constitutionnelle, ed. Cismonte e Pumonti, ler trimestre 1991, 64 p.

Unione di u Populu Corsu (UPC), Autonomia (document en supplément au journal Arriti), juin 1991, 215 p.

Presse, revues

"A Chajma", mensuel (A Chajma et CNTI)

"A Fiara", mensuel (ANC)

"ARRITI", Hebdomadaire (1JPC)

"AVVENE", Mensuel (STC)

"Corse-Matin", quotidien (édition locale de Nice-Matin)

"INSEE", direction régionale de Corse, "TFC 91 : tabl ?au de l'économie corse", éditée par

l'INSEE (D.R. Corse), 1991, 149 p.

"Cartoscope" de la Corse (20 cartes couplées à des données socio-économiques), édité par INSEE et OEC (Observatoire économique de la Corse), février 1992.

"Economie corse", revue trimestrielle éditée par INSEE et OEC.

"Dossiers d'économie corse" (dossiers à thèmes non périodiques), édités par INSEE et OEC.

"Kyrn magazine", hebdomadaire.

"La Corse", quotidien (édition locale du Provencal).

"Paese", hebdomadaire (MPA).

"U Ribombu", hebdomadaire (aCN).

Document nº 1692 : annexe au procès-verbal de la séance du 5 novembre 1990.

Assemblée nationale. Projet de loi portant statut de la Collectivité territoriale de Corse.

Document n ${ }^{\circ} 1706$ : annexe au procès-verbal du 15 novembre 1990.

Assemblée nationale, Rapport portant statut de la collectivité territoriale de Corse, par M José Rossi.

Assemblée nationale, Débats parlementaires du 22 novembre 1990 ; du 23 novembre 1990 ; du 24 novembre 1990. 
Document n 98. Annexe au procès-verbal de la séance du 26 novembre 1990.

Sénat. Projet de loi portant statut de la collectivité territoriale de la Corse.

Sénat. Débats parlementaires du 21 mars 1991 ; du 22 mars 1991; du 23 mars 1991.

\section{NOTES}

1. Propositions exposées dans l'ouvrage autonomia 91, voir bibliographie.

2. dans le texte du Secondu Quaternu de mars 1991, voir bibliographie.

3. La Corse, 9 septembre 1992

4. Dans son programme de 1991, Pruposti Pulitichi, voir bibliographie.

5. Aucun Etat n'envisageant sérieusement pour l'instant d'établir des relations sur un pied d'égalité avec les nationalistes corses en vertu du principe de droit international de non-ingérence dans les affaires d'un Etat, en l'occurrence français.

6. Si on établit une comparaison avec d'autres mouvements nationalistes en Europe tels les modérés irlandais du SDLP.

7. L'un des thèmes de réflexion en août 1992 était "la souveraineté dans l'Europe".

8. Seul parti nationaliste dont on a pu à cette occasion mesurer l'implantation

électorale, les autres étant associés dans une coalition qui rendait impossible une mesure, parti par parti.

9. Il suffit de se reporter à l'épisode de la constitution d'un "intergroupe" en 1983.

10. Refonte qui s'est traduite par la radiation de plus de 40000 inscrits ne satisfaisants pas aux nouveaux critères définis, le corps électoral étant passé de plus de 200000 inscrits en 1991 à moins de 158000 en 1992.

11. Par exemple, l'importance des moyens et pouvoirs donnés aux 6 grands offices régionaux qui sont dirigés par des membres du conseil exécutif.

12. Le critère étant la nécessité d'être une région dont le PIB par habitant est inférieur de $25 \%$ à la moyenne communautaire.

13. La Corse, 13 octobre 1992.

14. Le conseil régional d'Alsace a d'ailleurs agi dans le même sens dans sa motion du 30 octobre 1992.

15. Ainsi le "FARC" (Front armé révolutionnaire corse), mouvement clandestin qui semble très radical et qui est apparu à l'occasion de la revendication de plusieurs actions dans un communiqué à l'AFP le 19 novembre 1992. Ce groupe pourrait réunir des membres de différentes organisations clandestines dans un activisme beaucoup plus dur. 


\section{RÉSUMÉS}

A travers la division du mouvement national à l'égard du traité de Maastricht, l'auteur constate que l'image de l'Europe est assez floue en Corse. Le mouvement nationaliste se structure autour de la primauté des problèmes insulaires, et de la lutte pour le champ politique local. Ce retard dans la prise de conscience du fait européen risque de provoquer des radicalismes comme le montre la réorientation récente de la violence contre des cibles étrangères.

The disagreements within the national movement regarding Maastricht show that the image of Europe remains fuzzy in Corsica. The nationalist movement structures itself round local problems and struggles within the local political field. This delay in the recognition of Europe may increase radicalism as shown in the recent redirection of violence against foreign targets.

\section{INDEX}

Index géographique : Europe, France

Mots-clés : Nationalismes, séparatismes/ autonomismes

Thèmes : FLNC

\section{AUTEUR}

\section{CHRISTIAN LEFÉBVRE}

Doctorant en science politique à l'Université Paris-X-Nanterre 